IFUSP/P - 1276

August 1997

\title{
Heavy Quarks in Polarized Structure Functions
}

\author{
F.M. Steffenst \\ Instituto de Física \\ Universidade de São Paulo \\ C.P. 66318 \\ 05389-970, São Paulo, Brazil
}

\begin{abstract}
Quark mass effects are included in the calculation of polarized structure functions. In particular, the validity of fixed order perturbation theory and of massless evolution is studied in the framework of heavy quarks structure functions. The polarized version of the ACOT and MRRS interpolating schemes for the evolution of massive quarks distributions is also developed and studied. The different behaviours of the various approaches in $x$ and $Q^{2}$ are shown.
\end{abstract}

\section{Introduction}

There has been a great interest in ways to improve our knowledge of the heavy quark content of the structure functions, both theoretically [1, 2, 3, 4, 5, 6] and experimentally [7]. It is an important research subject for two main reasons: 1) The gluon distribution in the proton has been extensively studied through heavy quark lepton production; 2) There are theoretical uncertainties on how to determine heavy quark distributions in the threshold region and how to resum the large logs containing the quark masses.

So far, unpolarized electroproduction has been the major focus of attention. The reason behind this activity is the large charm component of $F_{2}$

\footnotetext{
${ }^{1}$ Email address:fsteffen@axpfep1.if.usp.br
} 
measured at HERA [7]. However, future experiments dedicated to measure $\Delta g$ [8], the polarized gluon distribution of the proton, will also probe the polarized charm distribution through open charm production. Hence the importance of a proper treatment of massive quarks in polarized Deep Inelastic Scattering (DIS). In earlier attempts to estimate the charm component of $g_{1 p}$, attention was mainly restricted to its anomalous contribution [9, 10, 11] and to the magnitude of $\Delta c$, the charm singlet axial charge [12, 13, 14. In particular, in Ref. [11] the $x$ dependence of the anomalous gluon contribution to heavy quarks was calculated. One of the conclusions of that work was that the inclusion of charm was quite relevant to the magnitude of $\Delta g$.

In any case, a detailed study of the $x$ dependence and of the evolution of polarized heavy quarks is still missing. There has been an early work of Buza et al. [4] where NLO corrections to the Photon Gluon Fussion (PGF) cross section is calculated and their $x$ dependence studied. However, as it will be clear soon, it is desirable to expresses the heavy quark component of $g_{1 p}$ in terms of heavy quark distributions. The main reason for this are the large logarithms which necessarily appear in the partonic cross sections. In this line, it is the purpose of this work to investigate how and when massive quarks can be treated as partons in polarized DIS.

In massless $Q C D$, the polarized structure function $g_{1 p}\left(x, Q^{2}\right)$ of the proton can be written as:

$$
\begin{aligned}
g_{1 p}\left(x, Q^{2}\right)= & \int_{x}^{1} \frac{d z}{z}\left\{C_{q}^{N S}\left(z, Q^{2}, \mu^{2}\right) \Delta q^{N S}\left(x / z, \mu^{2}\right)\right. \\
+ & \frac{1}{2 n_{f}} \sum_{k=1}^{n_{f}} e_{k}^{2}\left[\left(C_{q}^{S}\left(z, Q^{2}, \mu^{2}\right) \Delta \Sigma\left(x / z, \mu^{2}\right)\right.\right. \\
& \left.\left.+C_{g}\left(z, Q^{2}, \mu^{2}\right) \Delta g\left(x / z, \mu^{2}\right)\right]\right\},
\end{aligned}
$$

where the quark nonsinglet and singlet coefficient functions are

$$
C_{q}^{N S, S}\left(z, Q^{2} / \mu^{2}\right)=\delta(z-1)+\frac{\alpha_{s}\left(\mu^{2}\right)}{4 \pi} C_{q}^{(1), N S, S}\left(z, Q^{2} / \mu^{2}\right)+\ldots,
$$

while the gluon coefficient function is

$$
C_{g}\left(z, Q^{2} / \mu^{2}\right)=\frac{\alpha_{s}\left(\mu^{2}\right)}{4 \pi} C_{g}^{(1)}\left(z, Q^{2} / \mu^{2}\right)+\ldots
$$


and the sum runs over the light flavours. The singlet quark distribution is

$$
\Delta \Sigma\left(z, \mu^{2}\right)=\sum_{i=1}^{n_{f}} \Delta q_{i}\left(z, \mu^{2}\right),
$$

and the nonsinglet quark distribution is

$$
\Delta q_{N S}=\frac{1}{2} \sum_{i=1}^{n_{f}}\left(e_{i}^{2}-\frac{1}{n_{f}} \sum_{k=1}^{n_{f}} e_{k}^{2}\right) \Delta q_{i}\left(z, \mu^{2}\right) .
$$

We set the renormalization scale to be equal to the factorization scale, $\mu^{2}$. The $Q^{2} / \mu^{2}$ dependence appearing in Eqs. (2) and (3) is in the form of a logarithm. Given the $x$ dependence of the axial charges in Eqs. (四) and (5) at some scale $\mu^{2}$, we can calculate the structure function at any other scale $Q^{2}$ with the help of the perturbatively calculated coefficient functions and splitting functions. Basically, there are two ways to perform this evolution. First, we could fix $\mu^{2}$ and do the evolution via the logarithms appearing in the coefficient functions, a procedure which is called Fixed Order Perturbation Theory (FOPT) [20, 21]. This method becomes problematic when $Q^{2}>>\mu^{2}$ because large logarithms appear. Indeed, $C_{g}$ in Eq. (3) is proportional to $\alpha_{s}\left(\mu^{2}\right) \ln \left((1-z) Q^{2} / z \mu^{2}\right)$ and the LO approximation will be reliable as long as this term is much smaller than unity. On the other hand, in FOPT the structure functions are determined to any order once the relevant partonic cross section is calculated to that order. It is not necessary to know all the splitting functions either to evolve the parton distributions. Otherwise, we could set $\mu^{2}$ to the natural scale $Q^{2}$ and do the evolution via the DGLAP equations for $\Delta q^{N S}\left(z, Q^{2}\right), \Delta \Sigma\left(z, Q^{2}\right)$, and $\Delta g\left(z, Q^{2}\right)$. In other words, the logarithms in $Q^{2}$ would be resummed. This procedure would be the most adequate as in the case of FOPT we have always the problem of not knowing up to which $Q^{2}$ the evolution through the logarithms can be trusted.

The situation is more involved when we deal with massive quarks. To begin with, we have an extra scale in the coefficient functions, the heavy quark mass $m_{h}$. For $Q^{2}>>m_{h}^{2}$, the mass sole effect would be to replace $\mu^{2}$ by $m_{h}^{2}$ in the logarithms of the coefficient functions, which means that the quark mass is being used as the infrared regulator. The corresponding large logarithms can then be resummed in a similar fashion to the massless quarks. Indeed, this has been done to all orders of perturbation theory by Buza et al. [3] for the unpolarized structure functions. When $Q^{2} \sim m_{h}^{2}$, 
this resummation can not be done. It then seems natural to use FOPT to calculate the structure function in the threshold region, using the full mass dependent form for the coefficient function. However, it is desirable to have a scheme that interpolates the two regions. The recently proposed methods of Aivazis et al. (ACOT) [1] and of Martin et al. (MRRS) [6] do this interpolation. In the MRRS scheme, the relevant splitting functions are modified in the threshold region in order to incorporate mass effects in the evolution of the massive quark distributions. It means that a heavy quark distribution is introduced at threshold which is evolved with the help of the relevant mass modified splitting functions. Moreover, the gluon coefficient function is modified to avoid double counting. When $Q^{2}>>m_{h}^{2}$, their expressions are reduced to the massless convolution formulas. In the ACOT formalism, the splitting functions preserve their massless form but the coefficient functions retain their full dependence in $m_{h}^{2}$ to all orders of perturbation theory. Incidentally, we will see in the present work that the ACOT scheme is natural in polarized DIS.

It is the aim of this paper to study the evolution of the heavy quark component of $g_{1 p}$ using three different approaches. In section 2, the basics of the FOPT and of the massless evolution of $g_{1 p}^{h}\left(x, Q^{2}\right)$ are spelled out. In section 3 we calculate the modifications that the quark masses induce in the polarized splitting and coefficient functions. We then implement the interpolation schemes proposed in [1, 6]. The different methods for calculating $g_{1 p}^{h}\left(x, Q^{2}\right)$ are compared in section 4 , and their validity is discussed in section 5 .

\section{$2 g_{1 p}^{h}\left(x, Q^{2}\right)$ in FOPT and in Massless Evolu- tion}

In the absence of intrinsic heavy quarks in the proton, the dominant process for heavy quark production is PGF. This partonic cross section starts at order $\alpha_{s}$, and once it is known, one can in principle calculate the corresponding structure function at any scale. Hence, if at the scale $\mu^{2}$ the heavy quark distribution is zero, then the heavy quark component of the polarized structure function $g_{1 p}\left(x, Q^{2}\right)$ to order $\alpha_{s}$ is given by:

$$
g_{1 p}^{h}\left(x, Q^{2}\right)=e_{h}^{2} \frac{\alpha_{s}\left(\mu^{2}\right)}{4 \pi} \int_{a x}^{1} \frac{d z}{z} H_{g}^{(1)}\left(Q^{2}, m_{h}^{2}, z\right) \Delta g\left(\mu^{2}, x / z\right),
$$


where the PGF cross section for a massive particle, $H_{g}^{(1)}$, is 15, 16:

$$
H_{g}^{(1)}\left(Q^{2}, m_{h}^{2}, z\right)=T_{f}\left[4(2 z-1) \ln \left(\frac{1+s q}{1-s q}\right)+4(3-4 z) s q\right]
$$

with $T_{f}=1 / 2$ and

$$
s q=\sqrt{1-\frac{4 m_{h}^{2}}{(1-z) Q^{2}}} .
$$

The factor $a=1+4 m_{h}^{2} / Q^{2}$ in (6) ensures that the photon carries enough energy for the resolution of a heavy quark pair.

The questions to be answered are: given the scale $\mu^{2}$, how good is Eq. (6) to describe $g_{1 p}^{h}\left(x, Q^{2}\right)$ ? Up to which $x$ and $Q^{2}$ is Eq. (6) valid? In the absence of data, the answer to these questions should be in the comparison with different theoretical approaches. The main constraint to Eq. (6) is $\alpha_{s}\left(\mu^{2}\right) \ln \left(Q^{2} / m_{h}^{2}\right)<<1$, a condition that is fulfilled in the threshold region $\left(Q^{2} \sim m_{h}^{2}\right)$. However, when $Q^{2}>>m_{h}^{2}$ Eq. (7) is reduced to:

$$
H_{g}^{(1)}\left(Q^{2}, m_{h}^{2}, z\right)=T_{f}\left[4(2 z-1) \ln \left(\frac{1-z}{z} \frac{Q^{2}}{m_{h}^{2}}\right)+4(3-4 z)\right],
$$

and the large logarithms, mentioned in the introduction, appear. In that case, FOPT is not reliable any more. In an early study 20 it was argued that $Q^{2} / m_{h}^{2}<100$ would be a good approximation to FOPT. As we will see later, this is true only in the $x$ region studied in that work $(x>0.01)$. The discrepancy between FOPT and DGLAP evolution in fact grows as $x$ becomes smaller, becoming as different as $100 \%$ already at $x=10^{-4}$.

Of course, one could circumvent the problem with the large logarithms assuming massless evolution for heavy quarks over the entire $Q^{2}$ region. This approximation has been used in the literature for the unpolarized heavy quark distributions [17, 18. In such case, a heavy quark distribution function is introduced at a given scale, say $m_{h}^{2}$. Its value at any other scale is then predicted by the usual DGLAP equations. In this approach, the heavy quarks are resummed as massless particles and $g_{1 p}^{h}$ would be given by: 


$$
\begin{aligned}
g_{1 p}^{h}\left(x, Q^{2}\right) & =e_{h}^{2} \int_{x}^{1} \frac{d z}{z}\left[C_{q}\left(z, \alpha_{s}\left(Q^{2}\right)\right) \Delta h\left(x / z, Q^{2}\right)\right. \\
& \left.+\frac{\alpha_{s}\left(Q^{2}\right)}{4 \pi} C_{g}^{(1)}(z) \Delta g\left(x / z, Q^{2}\right)\right]
\end{aligned}
$$

with $\Delta h\left(z, m_{h}^{2}\right)=0$ and $C_{g}^{(1)}$ given by Eq. (9) taking $Q^{2}=m_{h}^{2}$. The massless quark coefficient at $O\left(\alpha_{s}\right)$ is

$$
\left.C_{q}\left(z, \alpha_{(} Q^{2}\right)\right)=\delta(z-1)+\frac{\alpha_{s}\left(Q^{2}\right)}{4 \pi} C_{q}^{(1)}(z)
$$

with [19, 20]

$$
\begin{aligned}
C_{q}^{(1)}(z) & =C_{F}\left[4\left(\frac{\ln (1-z)}{1-z}\right)_{+}-3\left(\frac{1}{1-z}\right)_{+}-2(1+z) \ln (1-z)\right. \\
& \left.-\frac{1+z^{2}}{1-z} \ln z+4+2 z+\delta(1-z)(-4 \zeta(2)-9)\right] .
\end{aligned}
$$

Although massless evolution would give the correct answer at $Q^{2}>>m_{h}^{2}$, it does not solve the problem of evolution of heavy quark distributions. To treat this problem, we apply the interpolating method of Refs. [1], 6] to the polarized case.

\section{$3 \quad g_{1 p}^{h}$ in an Interpolating Scheme}

As massless evolution for massive particles would be clearly wrong when $Q^{2} \sim m_{h}^{2}$, and FOPT would present problems at very large values of $Q^{2}$, it is desirable to have an scheme that interpolates between both. This procedure should reproduce Eq. (6) at the threshold and Eq. (10) when $Q^{2}>>m_{h}^{2}$. Despite the fact that the ACOT scheme [1] does this interpolation, in a recent work Martin et al. [6] developed an alternative scheme where such interpolation is also obtained. The main ingredients of their work are: 1) A heavy quark distribution is introduced at threshold, and its evolution is according to DGLAP type equations, with mass modified splitting functions; 2) The PGF cross section is modified to account for similar contributions 
coming from the evolution of the heavy quark distribution. This treatment ensures the absence of double counting, in the same spirit of the ACOT work.

As in our work we are interested in the polarized heavy quark component of the structure functions, we have to consider the mass modifications in the polarized splitting and coefficient functions in order to implement the MRRS scheme. We start computing the polarized gluon splitting function $\Delta P_{h g}$ in LO. The decay probability of the gluon into a polarized quark pair, where the quark carries a fraction $z$ of the gluon momentum is given by:

$$
d P_{h g}(z) d z=\frac{\alpha_{s}}{2 \pi} \Delta P_{h g} d z d l n\left(p_{\perp}^{2}+m_{h}^{2}\right)
$$

with the polarized splitting function being defined as:

$$
\Delta P_{h g}(z)=\frac{z(1-z)}{2} \frac{A}{p_{\perp}^{2}+m_{h}^{2}} .
$$

As usual, the four vectors will be defined in the Infinite Momentum Frame (IMF). The amplitude $A$ for the decay, where the parent gluon is taken to be always in a state of positive helicity, is:

$$
A_{ \pm+}=\operatorname{Tr}\left[\left(k_{h}+m_{h}\right) \gamma_{\mu}\left(k_{\bar{h}}-m_{h}\right) \gamma_{\nu} \frac{1 \pm \gamma_{5}}{2}\right] \epsilon_{+}^{* \mu} \epsilon_{+}^{\nu} .
$$

The gluon polarization vector is:

$$
\epsilon_{ \pm}=(0,0, \sqrt{1 / 2}, \pm i \sqrt{1 / 2})
$$

and the quark vectors are

$$
k_{h}=\left(z p+\frac{p_{\perp}^{2}+m_{h}^{2}}{2 z p}, z p, \vec{p}_{\perp}\right),
$$

with a similar expression for $k_{\bar{h}}$, just $z$ replaced by $1-z$. We also use the convention $\varepsilon^{0123}=+1$. After a straightforward calculation, we get:

$$
\begin{aligned}
P_{h+g+} & =T_{f} \frac{z(1-z)}{2} \frac{A_{++}}{\left(p_{\perp}^{2}+m_{h}^{2}\right)^{2}} \\
& =T_{f}\left(z^{2}+\frac{m_{h}^{2}}{p_{\perp}^{2}+m_{h}^{2}} z(1-z)\right), \\
P_{h-g+} & =T_{f}\left((1-z)^{2}+\frac{m_{h}^{2}}{p_{\perp}^{2}+m_{h}^{2}} z(1-z)\right) .
\end{aligned}
$$


The splitting function is then:

$$
\Delta P_{h g}=P_{h+g+}-P_{h-g+}=\frac{1}{2}\left(z^{2}-(1-z)^{2}\right),
$$

which is protected against mass effects. That means that a decay of a gluon into a massive quark pair does not produce any extra breaking of helicity. We can also get the unpolarized splitting function:

$$
P_{h g}=P_{h+g+}+P_{h-g+}=\frac{1}{2}\left(z^{2}+(1-z)^{2}\right)+\frac{m_{h}^{2}}{p_{\perp}^{2}+m_{h}^{2}} z(1-z),
$$

which agrees with the expression calculated in [6].

Our next step is to calculate the $\Delta P_{h h}$ splitting function: In this case,

$$
\Delta P_{h h}=C_{F} \frac{z(1-z)}{2} \frac{A}{p_{\perp}^{2}+m_{h}^{2}(1-z)^{2}},
$$

with

$$
A_{ \pm+}=\operatorname{Tr}\left[\left(k_{h}+m_{h}\right) \frac{1 \pm \gamma_{5}}{2} \gamma_{\nu}\left(k_{h}^{\prime}+m_{h}\right) \frac{1 \pm \gamma_{5}}{2} \gamma_{\mu}\right] \epsilon^{*} \cdot \epsilon .
$$

The scalar product of the gluon polarization vectors is $\delta^{i j}-k_{g}^{i} k_{g}^{j} /\left|\vec{k}_{g}\right|^{2}$, with $i, j=1,2,3$., and $k_{g}$ the gluon four momentum vector in the IMF. In the calculation, we took the initial quark to be always in a state of positive helicity. For the helicity conserving part, $A_{++}$, we get exactly the same result of the massless (and also unpolarized) case. For the helicity violating amplitude, we get $A_{-+}=-4 m_{h}^{2}$. It results in:

$$
P_{h-h+}=-2 C_{F} z(1-z) \frac{m_{h}^{2}}{p_{\perp}^{2}+m_{h}^{2}(1-z)^{2}}
$$

Hence,

$$
\Delta P_{h h}=C_{F}\left[\left(\frac{2}{1-z}\right)_{+}-1-z+\frac{3}{2} \delta(1-z)+2 z(1-z) \frac{m_{h}^{2}}{p_{\perp}^{2}+m_{h}^{2}(1-z)^{2}}\right]
$$

A similar calculation shows that 


$$
P_{g+h+}=C_{F}\left(\frac{1}{z}-z(1-z) \frac{m_{h}^{2}}{p_{\perp}^{2}+m_{h}^{2} z^{2}}\right)
$$

and

$$
P_{g-h+}=C_{F}\left(\frac{(1-z)^{2}}{z}-z(1-z) \frac{m_{h}^{2}}{p_{\perp}^{2}+m_{h}^{2} z^{2}}\right) .
$$

Thus $\Delta P_{g h}$ is also protected from mass effects. The same is not true for its unpolarized counterpart.

The last quantity to be calculated is the polarized partonic cross section, $\hat{g}_{1}^{(0)}$. It can be extracted from the Born approximation to the antisymmetric part of the partonic tensor.

The general structure for the antisymmetric part of the partonic tensor is:

$$
\hat{\omega}_{A}^{\mu \nu}=-\frac{m_{h}}{2 p . q} \varepsilon^{\mu \nu \alpha \beta} q_{\alpha}\left[s_{\beta} \hat{g}_{1}\left(z, Q^{2}\right)+\left(s_{\beta}-\frac{s . q}{p . q}\right) \hat{g}_{2}\left(z, Q^{2}\right)\right],
$$

where $p$ is the parton four momentum vector, $s$ its spin vector and $q$ the photon four momentum vector. If we define a general projector $P^{\mu \nu}=$ $\varepsilon^{\mu \nu \alpha \beta} A_{\alpha} B_{\beta}$, such that:

$$
P^{\mu \nu} \hat{\omega}_{\mu \nu}^{A}=-\frac{m_{h}}{2 p . q}(A . q B . s-A . s B . q) \hat{g}_{1}^{(0)}
$$

then

$$
\hat{g}_{1}^{(0)}=-\frac{2 p \cdot q}{m_{h}} \frac{1}{A . q B . s-A . s B \cdot q} P^{\mu \nu} \hat{\omega}_{\mu \nu}^{A} .
$$

In the Born approximation, the antisymmetric part of the partonic tensor is

$$
\hat{\omega}_{\mu \nu}^{A}=m_{h} \varepsilon_{\mu \nu \alpha \beta} s^{\alpha} q^{\beta} \delta\left(2 p \cdot q+q^{2}\right) .
$$

The use of Eq. (30) in Eq. (29) then renders a coefficient function in LO which is unmodified by the presence of quark masses:

$$
\hat{g}_{1}^{(0)}=\delta(z-1) .
$$

We see that in LO, the polarized splitting functions and the coefficient function are protected against mass corrections, with the exception of the 
$\Delta P_{h h}$ term. However, as shown in [6], any mass effect appearing in the LO expressions, will be felt only in NLO. This can be seen from the following. The general expression for the decay of a parton in LO would be:

$$
d P(z) d z=\frac{\alpha\left(p_{\perp}^{2}\right)}{2 \pi}\left\{\frac{f(z)}{p_{\perp}^{2}+m^{2}}+g(z) \frac{m^{2}}{\left(p_{\perp}^{2}+m^{2}\right)^{2}}\right\} d z d p_{\perp}^{2} .
$$

As is well known, if we integrate Eq. (32) $n$ times in $p_{\perp}^{2}$ using $m^{2}=0$, with a strong ordering in $p_{\perp}^{2}$, and sum over $n$, we obtain the $\left[\alpha\left(\mu^{2}\right) / \alpha\left(Q^{2}\right)\right]^{P}$ of the renormalization group equations, where $P$ is the splitting function. When $m^{2} \neq 0$, logarithms in $\alpha$ are systematically lost, which is a typical feature of NLO resummation. Besides this, the integrals of the mass terms in Eq. (32) are problematic, non analytical, and resummation is impossible. In the same way, any mass modification of the NLO splitting functions, would appear only in a NNLO study. With this in mind, we can then make a fully NLO evolution of a polarized heavy quark distribution using the same massless expressions, the only exception being the LO $\Delta P_{h h}$ term.

The next step is to define the massive PGF cross section which interpolates the massless coefficient, $C_{g}$ in Eq. (3), and the full massive coefficient, $H_{g}$ in Eq. (9). As noticed in Ref. [1], a part of $H_{g}$ has to be subtracted because when evolving the heavy quark distribution, the $\Delta P_{h g}$ splitting function will also create heavy pairs. In the massless evolution such a subtraction is already implicit in the absence of the $\ln \left(Q^{2}\right)$ term. Using the slow evolution variable, $\xi=\left(1+m_{h}^{2} / Q^{2}\right) x \equiv b x$, as defined by Brock [22], we write the heavy quark component as:

$$
\Delta h(x) \otimes \hat{g}_{1}^{(0)}(x)=\int_{\xi}^{1} \frac{d z}{z} \delta(z-1) \Delta h\left(\frac{\xi}{z}\right)=\Delta h(\xi) .
$$

Integrating the gluon splitting part of the LO DGLAP equation for $\Delta h$, we obtain:

$$
\Delta h(\xi)=\frac{1}{4 \pi} \int_{\xi}^{1} \frac{d z}{z} \int_{Q_{\min }^{2}}^{Q^{2}} d \ln Q^{\prime 2} \alpha_{s}\left(Q^{\prime 2}\right) \Delta g\left(z, Q^{\prime 2}\right) \frac{\Delta P_{h g}(\xi / z)}{2} .
$$

If we use $\alpha_{s} \Delta g$ as a scale invariant, then Eq. (34) is reduced to:

$$
\Delta h(\xi)=\frac{\alpha_{s}\left(Q^{2}\right)}{4 \pi} \ln \left(\frac{Q^{2}}{m_{h}^{2}}\right) \int_{b x}^{1} \frac{d z}{z} \Delta g\left(z, Q^{2}\right) \frac{\Delta P_{h g}(b x / z)}{2},
$$


where we use $m_{h}^{2}$ for the minimum value of the scale. According to the $\Delta P_{h g}$ splitting function, we should start to evolve the heavy quark distribution at $m_{h}^{2}$. This is explicit in Eqs. (14) and (35). And we actually implement this scale in our calculations. However, to avoid any unconsistency with the scale necessary for heavy quark to be resolved as a physical object (being probed by the photon), we will always calculate $g_{1 p}^{h}$ at scales where $Q^{2}>4 m_{h}^{2}$. We then incorporate into the heavy quark coefficient function a threshold factor of the form $\theta\left(1-4 m_{h}^{2} / Q^{2}\right)$. That means that below $4 m_{h}^{2}, g_{1 p}^{h}\left(x, Q^{2}\right)$ is given by PGF only. As the choice of the threshold factor is not unique, the calculation of the heavy quark structure functions is, in a sense, ill defined. We return to this point in the next section.

Finally, we write down the convolution formula for the heavy quark component of the polarized structure function:

$$
\begin{aligned}
g_{1 p}^{h}\left(x, Q^{2}\right) & =e_{h}^{2} \int_{b x}^{1} \frac{d z}{z} C_{q}\left(z, Q^{2}\right) \Delta h\left(\frac{b x}{z}, Q^{2}\right) \\
& +e_{h}^{2} \frac{\alpha_{s}\left(Q^{2}\right)}{4 \pi} \int_{a x}^{1} \frac{d z}{z} H_{g}^{(1)}\left(z, m_{h}^{2}, Q^{2}\right) \Delta g\left(\frac{x}{z}, Q^{2}\right) \\
& -e_{h}^{2} \frac{\alpha_{s}\left(Q^{2}\right)}{4 \pi} \ln \left(\frac{Q^{2}}{m_{h}^{2}}\right) \int_{b x}^{1} \frac{d z}{z} \frac{\Delta P_{h g}(z)}{2} \Delta g\left(\frac{b x}{z}, Q^{2}\right) .
\end{aligned}
$$

When we take $Q^{2}>>m_{h}^{2}$ the last two lines of Eq. (36) are reduced to the expression for massless quark production through PGF, and we get Eq. (10) corresponding to the massless case. We notice that this is an exact result.

In light of the resulting Eq. (36), it is instructive to analize the shortcomings of both MRRS and ACOT approaches for the calculation of the heavy quark structure functions. The most evident and serious problem with MRRS is the fact that one does not recover the massless, $\overline{M S}$ calculated, unpolarized gluon coefficient function when the limit $Q^{2}>>m_{h}^{2}$ is taken [6]. Hence, it can be hardly claimed that the defined heavy quark distributions are $\overline{M S}$ distributions. In the same line, $\overline{M S}$-like schemes are mass independent, meaning that evolution can not possibly depend on the mass of the quark, a requirement not fulfilled by the MRRS prescription. However, because of the mass independence of both the polarized quark coefficient function and the polarized splitting functions in LO (the exception being $\Delta P_{h h}$ but it has no numerical relevance - see next section), Eq. (36) 
is really the ACOT version for the calculation of the polarized heavy quark structure function. Hence, opposite to the unpolarized case, the polarized gluon coefficient function has the correct $\overline{M S}$ form in the limit $Q^{2}>>m_{h}^{2}$. In a sense, it can be said that the ACOT formulation is preferable over the MRRS formulation for $g_{1 p}\left(x, Q^{2}\right)$. In any case, the ACOT formulation also has formal drawbacks. Specifically, the gluonic contribution to the structure function, which is of order $\alpha_{s}$, enters together with the $\alpha_{s}^{0}$ quark contribution only, when the $\alpha_{s}$ quark corrections should also be considered. However, as stressed in the ACOT work [1], the $\alpha_{s}$ quark corrections are numerically insignificant when compared to the gluon contributions. In the particular case of the present work, the $\alpha_{s}$ quark corrections were estimated, and their contributions are negligible. Finally, both schemes have ambiguities related to the choice of the factorization scale $\mu^{2}$, when physical observables, like structure functions, should not depend on the chosen value for $\mu^{2}$. Fortunately, in practical calculations this dependence is not a real concern [2].

\section{Results}

We are now ready to calculate $g_{1 p}^{h}\left(x, Q^{2}\right)$. Although we will restrict ourselves to charm, the extension to other heavy quarks is immediate. We will use the NLO GRSV [23] "standard scenario" parametrizations for the gluon and quark singlet distributions as the input in our calculation. We made this choice because of the low scale where this parametrization is supposed to be valid, which allows us to evolve through charm threshold. The strategy used for evolution was the following. We first evolved the gluon and the singlet quark distributions from $0.34 \mathrm{GeV}^{2}$ to $m_{c}^{2}$, with 3 active flavours. From $m_{c}^{2}$ to $Q^{2}$, the charm quark distribution is obtained from the evolution of the singlet quark distribution with 4 flavours, using as input distributions $\Delta \Sigma\left(z, m_{c}^{2}\right)=0$ and the $\Delta g\left(z, m_{c}^{2}\right)$ obtained from the evolution from $0.34 \mathrm{GeV}^{2}$. The charm distribution at $Q^{2}$ is then $\Delta c\left(z, Q^{2}\right)=\Delta \Sigma\left(z, Q^{2}\right) / 4$. The gluon distribution at $Q^{2}$ is obtained from the singlet evolution of the gluon distribution with four active flavours, using as input distributions $\Delta \Sigma\left(z, m_{c}^{2}\right)$ and $\Delta g\left(z, m_{c}^{2}\right)$, which were obtained before when evolving from $0.34 \mathrm{GeV}^{2}$. Once we obtain the gluon and charm distributions at $Q^{2}$, we can use the various convolution formulas, Eq. (6) for the pure PGF evolution via FOPT, Eq. (36) for the improved MRRS method, and Eq. (10) for massless evolution. 
We use $m_{c}=1.5 \mathrm{GeV}$ for the charm mass, and $\Lambda_{Q C D}^{(4)}=0.2 \mathrm{GeV}$ in order to agree with GRSV. The coupling constant at any scale, from $\mu^{2}$ to $Q^{2}$, is given by the solution of the NLO equation for $\alpha_{s}$ :

$$
\frac{\alpha_{s}\left(Q^{2}\right)}{4 \pi}=\frac{1}{\beta_{0} \ln \left(Q^{2} / \Lambda_{Q C D}^{2}\right)}-\frac{\beta_{1}}{\beta_{0}^{3}} \frac{\ln \left(\ln \left(Q^{2} / \Lambda_{Q C D}^{2}\right)\right)}{\left(\ln \left(Q^{2} / \Lambda_{Q C D}^{2}\right)\right)^{2}} .
$$

We notice that the use of this equation at low values of $Q^{2}$ such as $0.34 \mathrm{GeV}^{2}$ can lead to difficulties. In this case, the use of the transcendental equation for $\alpha_{s}$ from which Eq. (37) is derived, would be more appropriate. However, to be consistent with the way the parametrizations were built, Eq. (37) have to be used.

In Fig. 11 we present the axial singlet charm distribution of the proton at $10 \mathrm{GeV}^{2}$. It is obtained as explained before, evolving the singlet distribution from $m_{c}^{2}$. This result shown is independent of the mass modified $\Delta P_{q q}$ splitting function. It means that even if $\Delta P_{q q}$ is the only term which receives any mass modification, it is also protected against mass terms for practical purposes. It is possible to fit the charm distribution as:

$$
\begin{aligned}
x \Delta c\left(x, 10 \mathrm{GeV}^{2}\right) & =x^{0.65}\left(-0.055(1-x)^{5}+0.206(1-x)^{6}\right) \\
& +x^{0.29}\left(-0.286(1-x)^{7}+0.261(1-x)^{8}\right) .
\end{aligned}
$$

The particular form for Eq. (38) was inspired by Brodsky et al. [24] considerations on the behaviour of the polarized distributions. The integral over $x$ of Eq. (38) is

$$
\Delta c\left(10 \mathrm{GeV}^{2}\right) \sim 2.6 \times 10^{-5},
$$

which is completely irrelevant. However, we notice that charm is allowed in a large portion of the existing data for $g_{1 p}$, meaning that the study of the $x$ dependence is relevant, mainly in the small $x$ region.

In Fig. 2 the charm component of $g_{1 p}$ at $10 \mathrm{GeV}^{2}$ is presented. The dotted line is the result from FOPT, through Eq. (6). In this case, $\mu^{2}=2.25 \mathrm{GeV}^{2}$ and $\alpha_{s}$ is calculated using $n_{f}=4$. The dashed line is the result from massless evolution. The continuous line is obtained with the help of Eq. (36), which is the scheme where low and high $Q^{2}$ are consistently incorporated. We see that down to $x \sim 4 \times 10^{-4}$, the three methods of calculating $g_{1 p}^{c}\left(x, Q^{2}\right)$ 


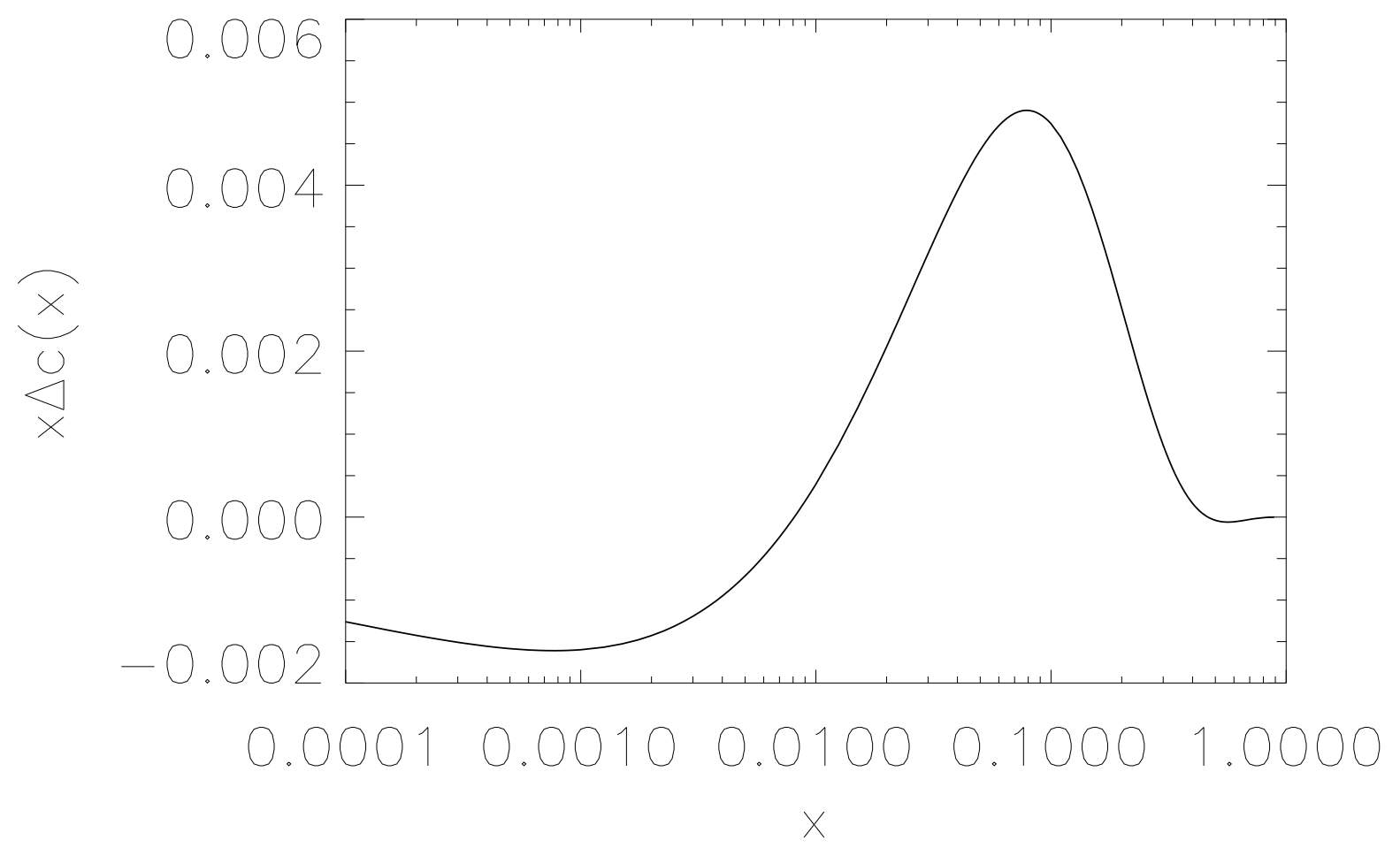

Figure 1: The $x$ dependence of the charm singlet axial charge at $10 \mathrm{GeV}^{2}$.

give very close results. However, as $x$ gets smaller FOPT fails to follow the other two methods. We think this failure happens because at $x=10^{-4}$, $\alpha_{s}\left(\mu^{2}\right) \ln \left((1-x) Q^{2} / x m_{c}^{2}\right) \sim 3$, and the NLO corrections calculated in [4] would need to be incorporated.

On the other hand, we plot in Fig. 3 the $x$ dependence of the pure PGF term, second line of Eq. (36) and the pure charm quark distribution, the first plus the third lines in Eq. (36). The dashed line is the PGF contribution and the continuous line the charm contribution. Comparing with Fig. 2, we see that the PGF alone would reproduce the FOPT calculation, which is a natural result as Eq. (6) is pure PGF. But the charm contribution is almost as large as the PGF, hence the discrepancy between the dotted and continuous line of Fig. 2. That happens because, as stated in the end of section 3, we choose not to add any ad hoc threshold factor in the charm coefficient functions. If we had added, for instance, the factor $f=$ 


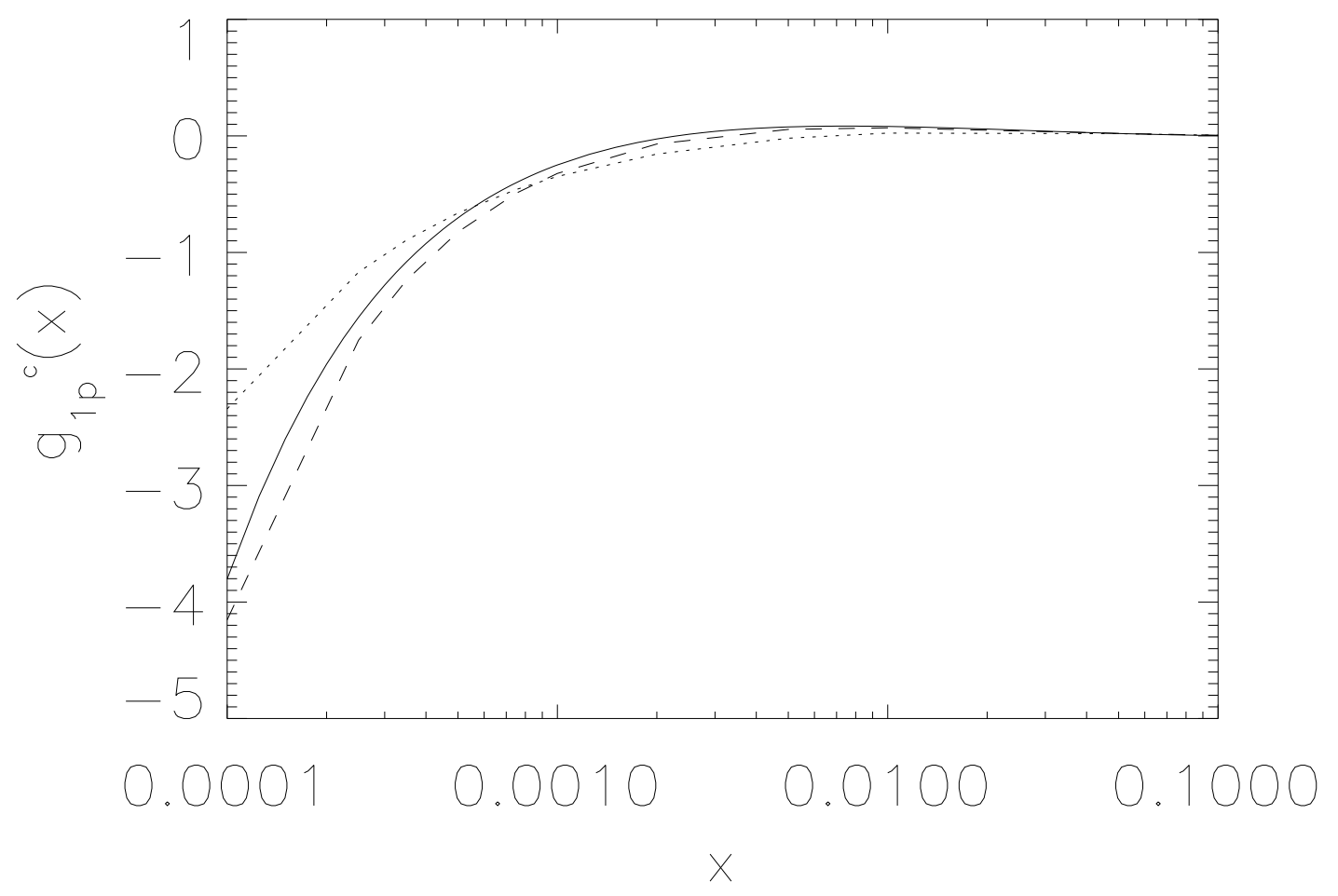

Figure 2: The charm component of $g_{1 p}\left(x, Q^{2}\right)$ at $10 \mathrm{GeV}^{2}$. The dotted line is the result from FOPT. The dashed line is the result from massless evolution and the continuous line represents Eq. (36).

$\left(1-4 m_{c}^{2} / Q^{2}\right) \theta\left(1-4 m_{c}^{2} / Q^{2}\right)$ as it is done in [6], the charm quark component of $g_{1 p}^{c}\left(x, Q^{2}\right)$ shown in Fig. 3 would be dramatically reduced. In this case, the line of Fig. 2 would be very close to the FOPT result. However, the factor $f$ is arbitrary and other choices for it would produce different contributions from the charm evolution. In this sense, the calculation of $g_{1 p}^{c}\left(x, Q^{2}\right)$ at threshold is ambiguous. In our case, we made the choice that if charm can be resolved, then it is given directly by Eq. (36). In other words, our threshold factor is the $\theta$ function only.

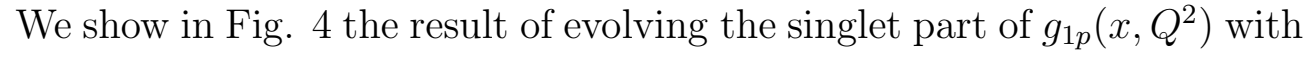
three and four flavours. The procedure used was as the same used before. For three flavours, we evolve directly from $0.34 \mathrm{GeV}^{2}$ to $10 \mathrm{GeV}^{2}$, using $n_{f}=3$ 


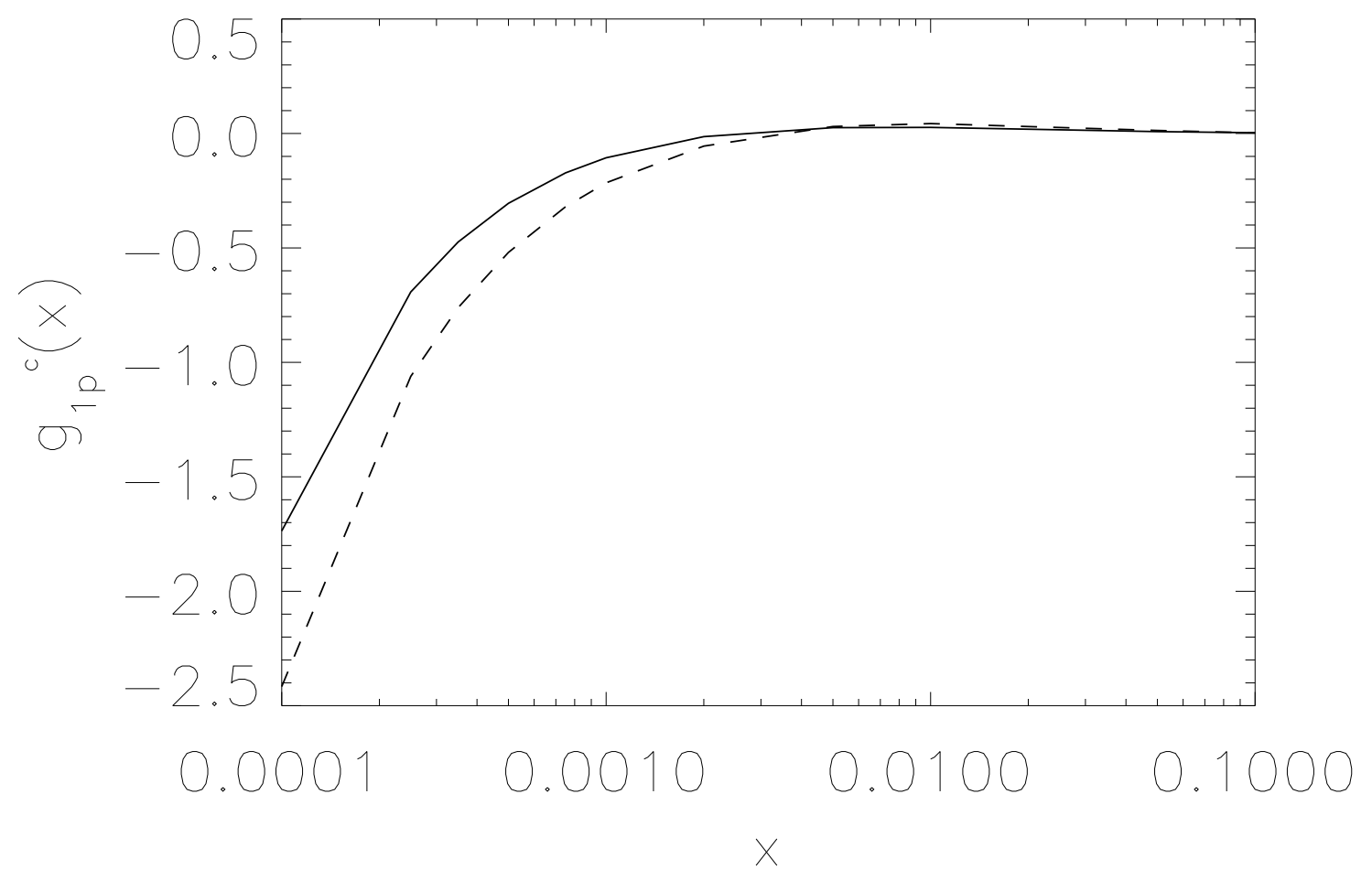

Figure 3: The continuous line is the charm component of Eq. (36) while the dashed line is the PGF term only. Both curves are calculated at $10 \mathrm{GeV}^{2}$.

and $\Lambda_{Q C D}^{(3)}=0.248 \mathrm{GeV}$. For the four flavours component, we evolve from $0.34 \mathrm{GeV}^{2}$ to $m_{c}^{2}$ in the three flavour theory and from $m_{c}^{2}$ to $10 \mathrm{GeV}^{2}$ in the four flavour theory. Of course, the procedure is not completely consistent as the GRSV parametrization was built using $n_{f}=3$ only. However, it is expected that at $m_{c}^{2}$ the parametrization is valid, meaning that the results shown here really point to the modifications that a fourth flavour would induce to $g_{1 p}^{S}\left(x, Q^{2}\right)$. Indeed, we see that at $x=10^{-4}$, a difference of the order of $30 \%$ is present.

Finally, Fig. 5 shows $g_{1 p}^{c}\left(x, Q^{2}\right)$ at $100 \mathrm{GeV}^{2}$. As expected, at this scale the improved equation for massive convolution reproduces exactly the massless approach. FOPT, however, presents the same sort of behaviour shown at $10 \mathrm{GeV}^{2}$. The reason for this behaviour is the same as explained before. 


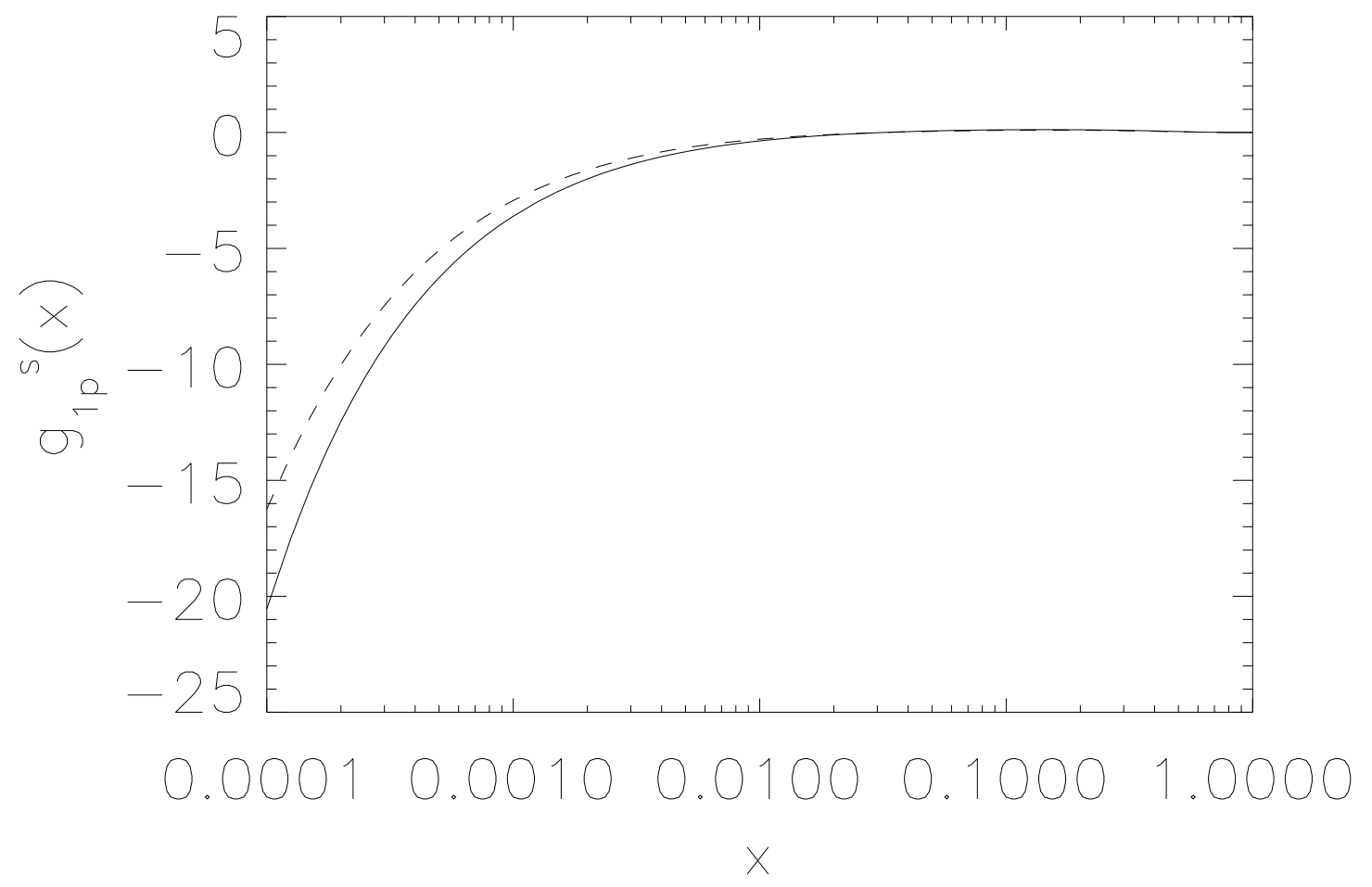

Figure 4: The singlet part of $g_{1 p}\left(x, Q^{2}\right)$. The continuous curve was evolved with 4 flavours, while the dashed curve was evolved with 3 flavours. Both curves were calculated at $10 \mathrm{GeV}^{2}$.

\section{Final Remarks and Discussion}

We have computed the charm component of the polarized structure function of the proton, $g_{1 p}\left(x, Q^{2}\right)$. We used three different methods in these calculations and it is remarkable that already at $10 \mathrm{GeV}^{2}$, massless evolution is a very good approximation. Of course, this conclusion is blurred by the umbiguous appearance of a possible threshold $f$ factor in the heavy quark coefficient functions. In any case, it is seen that FOPT is close to massless evolution down to $x=10^{-3}$, at least up to $Q^{2}=100 \mathrm{GeV}^{2}$. This conclusion complements an early observation [20] where it was argued that FOPT and DGLAP evolution were equally good. In truth, we see that when $x$ gets smaller, the large logarithms associated with the scale are enhanced by the 


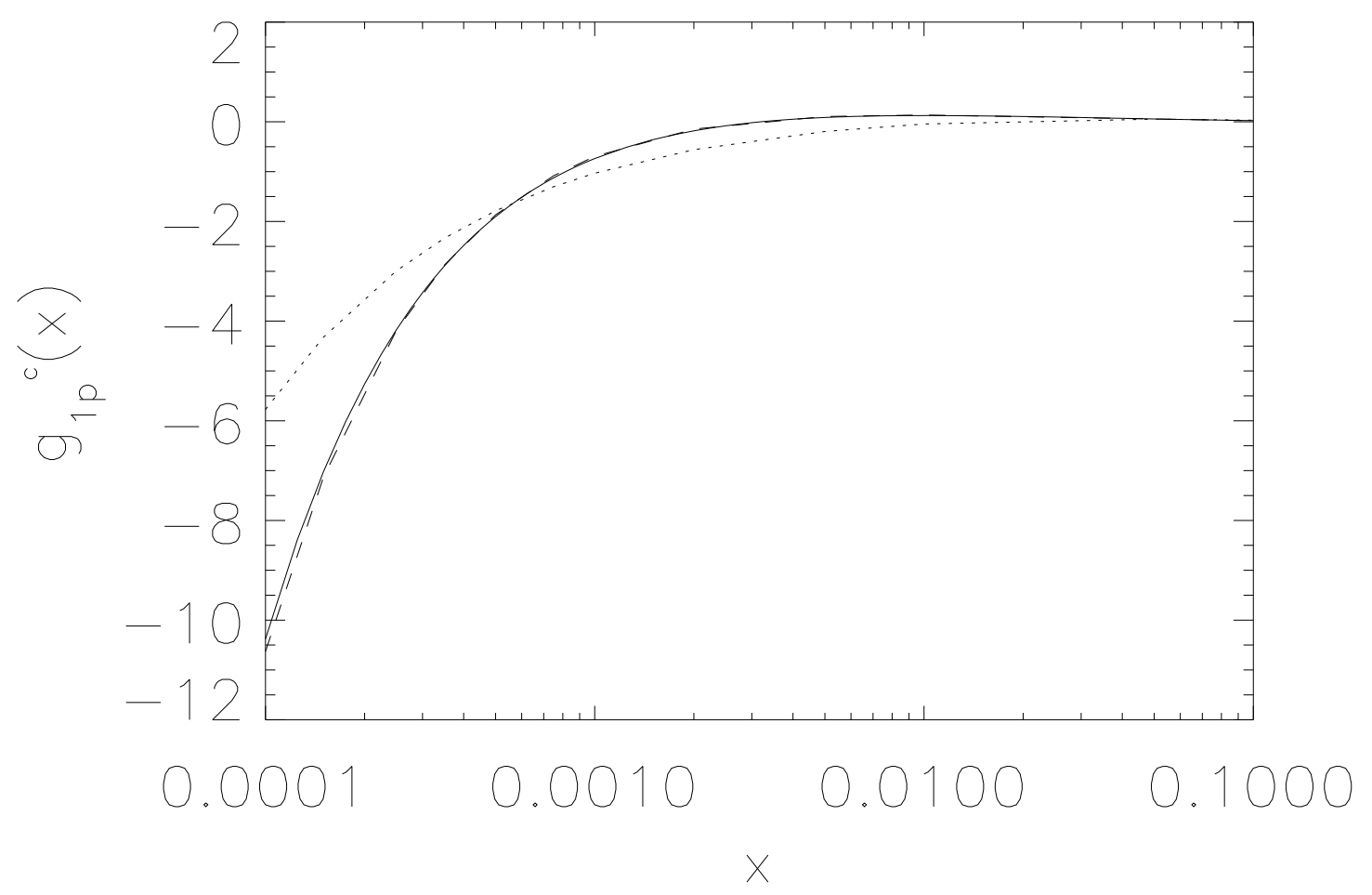

Figure 5: The same convention of Fig. 2, calculated now at $100 \mathrm{GeV}^{2}$.

$1 / x$ factors, something which does not happen in the usual evolution approach or in the improved method of Eq. (36) because the scale logarithms are absent. FOPT then breaks down. Moreover, we saw in Fig. 4 how important the inclusion of a heavy flavour can be for the singlet component of $g_{1 p}$, even if the amount of $\Delta c$ in the proton is small. Finally, when calculating the mass corrections to the splitting functions, we saw that they are protected against mass effects. Thus, effectively, Eq. (36) is the polarized version of the ACOT scheme [1] for heavy quarks.

I would like to thank to F. S. Navarra, W. Melnitchouk and A. W. Thomas for the many discussions on the subjects covered in this work as well as for a careful reading of this manuscript. I also would like to thank to T. Weigl for the basic code for NLO evolution. Furthermore, I profited from the access 
to the computer system of the Centre for the Subatomic Structure of Matter

in Adelaide, Australia. Finally, I would like to thank the Instituto de Física Teórica (IFT) for their support and hospitality, and where part of this work was completed. This work was supported by FAPESP (Brazil).

\section{References}

[1] M. A. G. Aivazis, J. C. Collins, F. I. Olness and W.-K. Tung, Phys. Rev. D50 (1994) 3102; W.-K. Tung, hep-ph 9706480.

[2] F. I. Olness and R. J. Scalise, hep-ph/9707459.

[3] M. Buza, Y. Matiounine, J. Smith and W. L. van Neerven, hep$\mathrm{ph} / 9612398$.

[4] M. Buza, Y. Matiounine, J. Smith and W. L. van Neerven, Nucl. Phys. B485 (1997) 420.

[5] J. Smith, hep-ph/9708212.

[6] A. D. Martin, R. G. Roberts, M. G. Ryskin and W. J. Stirling, hep$\mathrm{ph} / 9612449$.

[7] C. Adloff et al, Z. Phys. C72 (1996) 593; M. Derrick et al.,Phys. Lett. B349 (1995) 225.

[8] G. K. Mallot, in behalf of the COMPASS collaboration, hep-ex/9611016.

[9] J. Ellis, M. Karliner and C. T. Sachrajda, Phys. Lett. B231 (1989) 497.

[10] S. D. Bass, B. L. Ioffe, N. N. Nikolaev and A. W. Thomas, J. Moscow. Phys. Soc. 1 (1991) 317.

[11] F. M. Steffens and A. W. Thomas, Phys. Rev. D53 (1996) 1191.

[12] A. V. Manohar, Phys. Lett. B242 (1990) 94.

[13] G. Altarelli and B. Lampe, Z. Phys. C47 (1990) 315.

[14] S. D. Bass, R. J. Crewther, F. M. Steffens and A. W. Thomas, hepph/9701213. 
[15] D. A. Watson, Z. Phys C12 (1982) 123.

[16] M. Glück, E. Reya and W. Vogelsang, Nucl. Phys. B351 (1991) 579.

[17] A. D. Martin, R. G. Roberts and W. J. Stirling, Phys. Rev. D50 (1994) 6734.

[18] H. L. Lai et al. (CTEQ), Phys. Rev. D51 (1995) 4763.

[19] J. Kodaira, S. Matsuda, K. Susaki and T. Kematsu, Nucl. Phys. B159 (1979) 99; J. Kodaira, S. Matsuda, T. Muta, K. Susaki and T. Kematsu, Phys. Rev. D20 (1979) 627.

[20] E. B. Zijlstra and W. L. van Neerven, Nucl. Phys. B417 (1994) 61; Erratum Nucl. Phys. B426 (1994) 245.

[21] M. Glück, E. Reya and M. Stratmann, Nucl. Phys. B422 (1994) 37.

[22] R. Brock, Phys. Rev. Lett. 44 (1980) 1027.

[23] M. Glück, E. Reya, M. Stratmann and W. Vogelsang, Phys. Rev. D53 (1996) 4775.

[24] S. J. Brodsky, M. Burkardt and I. Schmidt, Nucl. Phys. B441 (1995) 197. 